\title{
Experimental Study on the Application of the Michelson Interferometer Yiying Lyu ${ }^{a}$, Lei Ma ${ }^{b}$ and Qili Yang ${ }^{c}$

\author{
School of Opto-Electronic Engineering, Zaozhuang University, Zaozhuang 277160, China

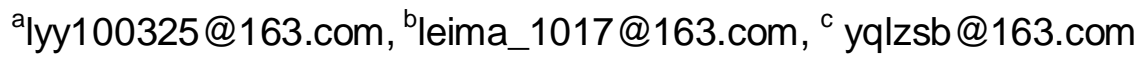

Keywords: Michelson interferometer; Applications; Teaching reform

\begin{abstract}
Traditionally, the optics experiment of tradition takes the lead of the authenticating experiment and goes short of interesting and activity, meanwhile it is very unfavorable to the expansion of students' creative ability. In this paper, applications of Michelson interferometer in laser wavelength measurement and surface roughness measurement are introduced to extend basic experiments for Michelson interferometer. The wavelength and properties of two kinds of lasers are obtained; and the variation of the interference pattern with the flatness of metal surface is shown as well.
\end{abstract}

\section{Introduction}

Michelson interferometer [1,2] is a common precision optical instrument in university laboratory, which uses the method of dividing amplitude to produce two coherent light beams. Measurements can be made by observing the changes in the fringe of the interference patterns. Researches on the application of Michelson interferometer can improve the teaching effect, the students' experimental operation skills, and thus train the application innovation ability of the students [3-4]. Researches about thickness and refractive index measurement by Michelson interferometer has been reported in some papers [5-7]. Experiments of Michelson interferometer on the wavelength measurement of two kinds of laser and detecting of surface flatness for three pieces of metal sheets are introduced in this paper. And the results shown that the measurement is precise and visualized when Michael interferometer is used.

\section{Experiment principles}

Principle of Michael interferometer. The working principle of the Michelson interferometer is shown in Figure 1[8]. $\mathrm{S}$ is a laser light source, the plate G1 and G2 are glass plates of equal thickness, and G1 was coated with a layer of semi-permeable membrane. The beam is reflected and refracted on the semi-permeable membrane, and is divided into two perpendicular beams of light which are refracted light (1) and reflected light (2). The light (1) goes through plate G2, comes into the mirror M1 and then reflected back to G2, and finally get to screen p; the light (2)incidents to mirror M2, reflected and then goes through G1, finally get to screen p. These two beams meet the coherence conditions, and the interference fringes are displayed on the screen $\mathrm{p}$. If the plane mirror M1 and M2 are perpendicular to each other, meet the inclinable interference conditions (which can be added to an expanding beam mirror to provide light incident at different angles); and If there is a small angle between the M1 and the M2, it belongs to the wedge interference device. And some physical quantities can be measured by observing the change of interference fringes $[9,10]$.

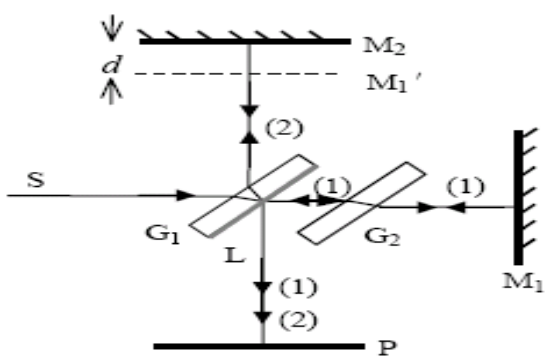

Figure 1. The schematic diagram of the Michelson interferometer 
Principle of small quantity measurement by equal inclination interference. When the moving mirror of the Michelson interferometer moves half a wavelength each time, an interference ring fringe can be observed or disappeared on the projection screen. And the micro displacement can be calculated by recording the number of interference fringes, the relation is shown as follow:

$$
d=N \frac{\lambda}{2}
$$

Here $\mathrm{d}$ is the displacement, $\mathrm{N}$ is the number of observed interference fringes that is produced or disappeared, and $\lambda$ is the wavelength of the light source.

Characteristic analysis of He- Ne laser and semiconductor laser. He- Ne laser is a gas laser with neutral atomic gas helium and neon as working materials [11], output continuous laser with continuous excitation. It has the advantages of good monochromatic property, good coherence, high brightness and strong directivity, and can be used as a point light source. Its disadvantage is power instability, complicated maintenance. Thus its use is gradually decreasing in the University's optical laboratory.

A semiconductor laser uses semiconductor material as working substance to produce a laser[12]. It has the advantages of high efficiency, small size, light weight, simple structure, good reliability, long service life and low price, and the defect is Performance instability.

Principle of flatness detection with interference fringes. The intensity of two coherent beams decreases and the brightness is smaller when the surface of the material is rough $[13,14]$, because of the lower reflectivity. The result is the blurred image of interference fringe. That is to say the smoother the material surface is, the clearer the interference fringe image is. Therefore, the smoothness of the material surface can be judged by the quality of the interference fringes.

\section{Experimental results and Discussion}

The results are measured with a interferometer (KF-WSM200, Hangzhou precision flying company). The light sources are semiconductor laser (LP-3) and a standard he- ne laser for laboratory use.

Experimental Data and Result Analysis of Laser Wavelength Measurement. Table 1 shows the data of wavelength measurement for he-ne laser, and table 2 is for semiconductor laser.

Table 1. Data of he-ne laser wavelength

\begin{tabular}{|l|l|l|l|l|l|l|}
\hline $\mathrm{d}_{\mathrm{i}}[\mathrm{mm}]$ & $\begin{array}{l}60.6114 \\
8\end{array}$ & 60.62775 & $\begin{array}{l}60.6437 \\
5\end{array}$ & $\begin{array}{l}60.6603 \\
8\end{array}$ & 60.67662 & 60.69243 \\
\hline $\mathrm{d}_{\mathrm{i}+6}[\mathrm{~mm}$ & 60.7082 & 60.72428 & 60.7406 & 60.7564 & 60.77268 & 60.78869 \\
] & 7 & & 8 & 9 & & \\
\hline$\Delta \mathrm{d}[\mathrm{mm}]$ & 0.09679 & 0.09653 & 0.09639 & 0.09611 & 0.09606 & 0.09626 \\
\hline
\end{tabular}

Table 1 shows the data of measurement for he-ne laser wavelength. The calculated wavelength is $643.3 \mathrm{~nm}$ according to formula (1). The reference wavelength of He-Ne laser is $632.8 \mathrm{~nm}$ according to relevant literature. Thus the relative error was calculated to be $1.6 \%$, and the measurement accuracy is $2.7 \times 10^{-4}$. It can be seen that the result of using Michelson interferometer to measure the wavelength of laser source is very accurate, and the performance of the laser is very stable throughout the test.

Table 2 data of semiconductor laser

(a) The first measurement data of semiconductor laser wavelength

\begin{tabular}{|l|l|l|l|l|l|l|}
\hline $\mathrm{d}_{\mathrm{i}}[\mathrm{mm}]$ & 60.82082 & 60.83814 & 60.85470 & 60.8715 & 60.88841 & 60.90473 \\
\hline $\mathrm{d}_{\mathrm{i}+3}[\mathrm{~mm}]$ & 60.87144 & 60.88786 & 60.90455 & 60.92151 & 60.93839 & 60.95575 \\
\hline$\Delta \mathrm{d}[\mathrm{mm}]$ & 0.05062 & 0.04972 & 0.04985 & 0.05001 & 0.04998 & 0.05102 \\
\hline
\end{tabular}

(b) The second measurement data of semiconductor laser wavelength

\begin{tabular}{|l|l|l|l|l|l|l|}
\hline $\mathrm{d}_{\mathrm{i}}[\mathrm{mm}]$ & 61.79603 & 61.81297 & 61.83052 & 61.84817 & 61.86502 & 61.88284 \\
\hline $\mathrm{d}_{\mathrm{i}+3}[\mathrm{~mm}]$ & 61.84753 & 61.86418 & 61.88145 & 61.90082 & 61.91569 & 61.93351 \\
\hline$\Delta \mathrm{d}[\mathrm{mm}]$ & 0.0515 & 0.05121 & 0.05093 & 0.05265 & 0.05067 & 0.05203 \\
\hline
\end{tabular}


(c) The third measurement data of semiconductor laser wavelength

\begin{tabular}{|l|l|l|l|l|l|l|}
\hline $\mathrm{d}_{\mathrm{i}}[\mathrm{mm}]$ & 62.40861 & 62.42546 & 62.44260 & 62.45988 & 62.47691 & 62.49447 \\
\hline $\mathrm{d}_{\mathrm{i}+3}[\mathrm{~mm}]$ & 62.46009 & 62.47708 & 62.49425 & 62.51148 & 62.52814 & 62.54612 \\
\hline$\Delta \mathrm{d}[\mathrm{mm}]$ & 0.05148 & 0.05162 & 0.05165 & 0.05160 & 0.05123 & 0.05165 \\
\hline
\end{tabular}

Table 2 shows the data of measurement for semiconductor laser. As the semiconductor laser has no standard wavelength to compare, three times measurements was made to ensure accuracy. The wavelengths obtained from this three measurements are $669.3 \mathrm{~nm}, 686.6 \mathrm{~nm}$ and $687.1 \mathrm{~nm}$ respectively, as it shows at Tab. 2 And the results of this three times measurements are quite different, the maximum deviation can be reached up to $2.6 \%$. That is because semiconductor lasers exhibit noticeable performance changes as temperature changes, the temperature will rise as time goes on. For this problem, a thermostat can be used in the experiment to stabilize the performance of semiconductor lasers.

The result of flatness detection of metal surface. Figure 2 shows that the interference fringes are clear and complete when the metal sheet which is used as a reflector has the highest surface smoothness, as it shown in Fig. 2 (a). Besides the intensity distribution of the fringes is uniform and the stripes are continuous. Fig. 2 (b) shows that for rougher surfaces, although circular interference fringes appeared, but some fringes broke, and some area that should have been the bright pattern has become dark, at the same time the light intensity distribution is uneven. Finally, the interference fringes formed by the metal sheet with the lowest flatness are shown in Fig. 2 (c). The brightness of the interference fringes becomes very weak and distribution is uneven. Therefore the Conclusion is that the flatness of the metal surface can be judged by contrasting the interference fringes with that of the standard.
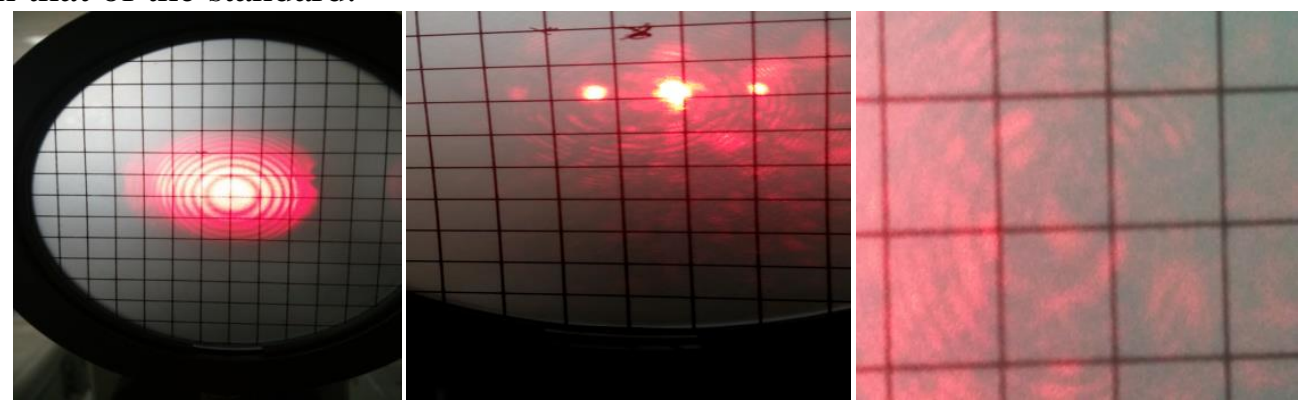

Figure 2. Interference fringes formed by metal surfaces with different roughness level.

\section{Summary}

Michelson interferometer is one of the most common precision optical instruments in the optical laboratory of the University, which is widely used in the field of measurement. In teaching practice, students can be guided to expand its applications, carry out innovating experiments and application-oriented experiments, so as to improve their psychologically tolerance, to inspire personality, to raise creative ability and to put theories into practice, thus the teaching effect can be improved greatly.

\section{Acknowledgements}

This work is supported by national natural science fund project (No. 11347158); Science and technology plan project of Shandong higher education institution (No. J15LN36); Science and technology program of Zaozhuang (No. 2016GX35); General Physics (Applied) high-quality curriculum project of Zaozhuang University (No. 233021502). 


\section{References}

[1] Has I, Miclaus S, Has A: An alternative light path analysis in Michelson's interferometer experiment, Physics Essays, 2010, 23(2)248-257.

[2] Tian, Z., S. S. Yam, and H. P. Loock: Refractive index sensor based on an abrupt taper Michelson interferometer in a single-mode fiber, Optics Letters 33.10(2008):1105.

[3] Information on https://wenku.baidu.com/view/8d4bd814866fb84ae45c8d47.html

[4] Ping Zhang, Chengxia Hou, Jinpan Song: Study and exploration of comprehensive design-oriented experiment teaching: Extended application of Michael interferometer, Experimental technology and management, 2011 , 28 (8):157-159( In Chinese)

[5] Xiang Z Y, Luo H, Ting-Ting M A, et al: Research on a Series of Development Experiments Based on Michelson Interferometer, Physical Experiment of College, 2016.

[6] Smith L M, Dobson C C: Absolute displacement measurement using modulation of the spectrum of white light in a Michelson interferometer, Appl Opt, 1989, 28(16):3339-3342.

[7] Li Jiang: Measurement of holographic film thickness using Michelson interferometer, Applied Optics, 2006,27(3)250-253.( In Chinese)

[8] Medhat M, El-Zaiat S Y, Omar M F, et al: Refraction and dispersion measurement using dispersive Michelson interferometer, Optics Communications, 2017, 393:275-283.

[9] Liu X, Wang C, Shang Y, et al: Distributed acoustic sensing with Michelson interferometer demodulation, Photon sensors: English Edition, 2017(3):193-198.

[10] Biao Yan , Hai Wang, Chunlai Yang, Li Wen: A calibration mechanism based on the principles of the Michelson interferometer micro-thrust test device, World Scientific.

[11] Linke R. Funktionsweise eines: Michelson-Interferometers// Ein Michelson-Interferometer aus LEGO®-Bausteinen. Springer Fachmedien Wiesbaden, 2017.

[12] Liu W J, Gao R X, Sun Z H, et al: Michelson Interference Applied to Measure Ultrashort Pulse, Physical Experiment of College, 2017.

[13] Li W, Chen L, Zhu J, et al: Apparatus for detecting the flatness of wafer and the method thereof, Clinical Biomechanics, 2015, 23(10):1209-19.

[14] Hatsuda M, Shimizu T: Surface inspection method and surface inspection apparatus, US, 2015.

[15] Information on https://wenku.baidu.com/view/270af77dcc22bcd127ff0cd7.html 